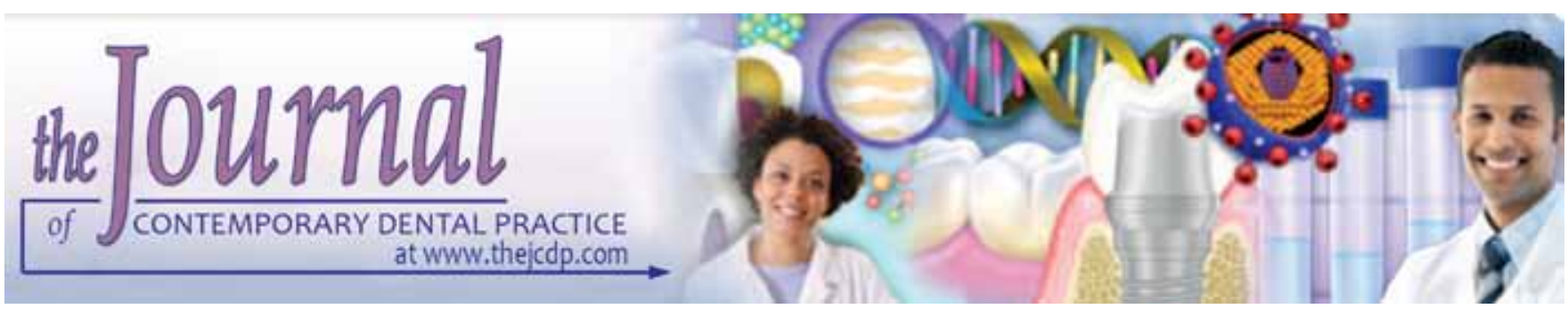

\title{
Influence of Nicotine and Cotinine Impregnation on the First Step of Periodontal Regeneration: Clot Stabilization
}

Shelon Cristina Souza Pinto, Fabio Renato Manzolli Leite, Lucas Amaral Fontanari, Rodrigo Cavassim, Amauri Antiquera Leite, Matheus Coelho Bandéca, Alvaro Henrique Borges, José Eduardo Cezar Sampaio

\begin{abstract}
This study analyzes the clot stabilization on root surfaces of teeth impregnated with cotinine and nicotine and the influence of the scaling in the adhesion of blood components, observing the influence of new exposition to nicotine and/or cotinine after scaling. Fifteen human teeth extracted due to periodontal disease of non-smokers patients were selected and manually scaled. Four dentin blocks were obtained from each tooth $(n=60)$. Samples received blood application or reimpregnation with nicotine and/or cotinine, depending on the groups. Group 1: PBS immersion + root scaling + blood; group 2: nicotine + root scaling + blood; group 3: nicotine + root scaling + nicotine reapplication + blood; group 4: cotinine + root scaling + blood; group 5: cotinine + root scaling + cotinine reapplication+ blood; group 6: nicotine and cotinine + root scaling + nicotine and cotinine + blood. Samples were kept in $2 \mathrm{ml}$ of each substance for 24 hours. Each group received a blood drop and was analyzed by SEM. The higher amount of blood components was present in teeth exposed to cotinine and the groups submitted to scaling and blood application in comparison with groups that received reapplication of toxic substances after scaling. The greater toxic effect on root dentin surface was after the exposure to nicotine and cotinine. Results suggest that periodontal healing may be delayed in smokers due to the direct inhibition of clot stabilization on the root surface when nicotine and cotinine are present concomitantly.
\end{abstract}

Keywords: Clot stabilization, Periodontal regeneration, Nicotine, Cotinine.

How to cite this article: Pinto SCS, Leite FRM, Fontanari LA, Cavassim R, Leite AA, Bandéca MC, Borges AH, Sampaio JEC. Influence of Nicotine and Cotinine Impregnation on the First Step of Periodontal Regeneration: Clot Stabilization. J Contemp Dent Pract 2013;14(6):1044-1048.

Source of support: This work was supported by CAPES (Coordenação de Aperfeiçoamento de Pessoal de Nível Superior).

Conflict of interest: None declared

\section{INTRODUCTION}

The organization of a clot and its stability along the root surface is one of the first steps to promote periodontal regeneration. ${ }^{1-4}$ Collagen fibers from dentin and cementum allow the fibrin network stabilization that is an essential condition to slow the long junctional epithelium formation in periodontal repair. ${ }^{5}$

During the first step to regeneration, smokers can be harmed due to impregnation of toxic substances on the teeth. ${ }^{6}$ The main toxic substance of the cigarette is nicotine. Some adverse effects of this substance are related to lack of platelet adhesiveness, which causes microclots and decreases microvascular perfusion. ${ }^{8}$ Another effect of nicotine is the decrease in red blood cells, fibroblasts and macrophages proliferation, impairing the healing process in surgical sites of smokers. ${ }^{9}$

Cotinine the major metabolite of nicotine is considered an accurate indicator of current smoking or exposure to smoke. Nicotine presents a short half-life in the blood, approximately 2 hours; in contrast, cotinine presents a longer serum half-life of approximately 19 hours. ${ }^{6,7}$

According to literature, nicotine and cotinine present in the root surface of patients who stopped smoking after the periodontal therapy can be eliminated in some cases, however, these toxic substances can still be found on root surface after scaling in a large number of patients with potential to modify cell functions, fibroblast growth and insertion in periodontal wound repair. ${ }^{6}$

The harm caused by nicotine and cotinine during the periodontal regeneration may increase in smoker patients when they keep the habit after the periodontal treatment, presenting new deposits of toxic substances on the root surface, however the delayed healing due to new exposition to nicotine and cotinine after periodontal treatment has not been evaluated. Studies have been showing the influence of tobacco smoking on periodontal healing, including fibroblasts colonization; ${ }^{5,6,9-11}$ however, the first step of the healing process consisting in clot stabilization has never been evaluated. 
Thus, the aim of this study was to analyze the clot stabilization on root surfaces of teeth impregnated with cotinine and nicotine and the influence of the scaling in the adhesion of blood components, evaluating the influence of new exposition to nicotine and/or cotinine after scaling.

\section{MATERIALS AND METHODS}

This study was approved by the Sao Paulo State University -Araraquara Dental School (UNESP - FOAr) Ethical Committee (23/2008).

\section{Sample Preparation}

Fifteen human single- and multirooted teeth extracted due to severe periodontal disease of nonsmoker patients were used in this investigation. Teeth were obtained in the Human Teeth Bank of the Sao Paulo State University - Araraquara Dental School.

A high-speed cylindrical diamond bur (KG Sorensen, Barueri, SP, Brazil) under copious irrigation was used in order to make two parallel grooves $0.5 \mathrm{~mm}$ deep on the proximal root surfaces of each tooth (mesial and distal): one at the cementoenamel junction and the other $4 \mathrm{~mm}$ apical to the first. The area between the two grooves was flattened with the same bur. ${ }^{5}$

Teeth samples were impregnated in PBS-solution containing nicotine $(1 \mathrm{mg} / \mathrm{ml})$ and/or cotinine $(1 \mu \mathrm{g} / \mathrm{ml})$ for 24 hours. The delimited area was scaled with Gracey Curettes 5/6 (Hu-Friedy) through 50 cervical-occusal traction movements by the same operator to remove the contaminated cement and dentin.

The roots were cross cut in the first groove, in order to remove the crown. Four dentin blocks, approximately $2 \times 2 \mathrm{~mm}$, were obtained from each tooth, two from the mesial and two from the distal surface. Thus, 60 samples were obtained from the 15 teeth. Immediately, some samples received blood application or reimpregnation with nicotine and/or cotinine, depending on the groups as described below.

\section{Impregnation with Nicotine and Cotinine}

Nicotine (N-3876-Sigma Chemical Products, USA) and cotinine (C-5923-Sigma Products, USA) solutions were prepared by dilution in PBS (phosphate buffered solution) (Nociti et al, 2001; Breivik, Gundersen, Gjermo, Hörsten, and Opstad, 2009). This study tested nicotine and cotinine concentrations based on their concentration in saliva of smoking patients: nicotine $-1 \mathrm{mg} / \mathrm{ml}$ and cotinine $-1 \mu \mathrm{g} /$ $\mathrm{ml}(10,13)$. Samples were kept in $2 \mathrm{ml}$ of each substance for 24 hours.

\section{Experimental Groups}

- Group 1: PBS immersion + root scaling + blood$(\mathrm{n}=10)$.

- Group 2: Nicotine + root scaling + blood - $(\mathrm{n}=10)$.

- Group 3: Nicotine + root scaling + nicotine reapplication + blood - $(\mathrm{n}=10)$.

- Group 4: Cotinine + root scaling + blood $-(\mathrm{n}=10)$.

- Group 5: Cotinine + root scaling + cotinine reapplication + blood - $(\mathrm{n}=10)$.

- Group 6: Nicotine and cotinine + root scaling + nicotine and cotinine + blood $-(\mathrm{n}=10)$.

\section{Blood Application}

Fresh human whole peripheral blood from a healthy male donor was applied to the external root surface of 10 specimens of each group. Clot was allowed to maturate on root surface for 20 minutes in a humidified chamber at $37^{\circ} \mathrm{C} ., 5,12$ Samples were then rinsed three times for 5 minutes in PBS. Washes and rinses of the root samples were performed in small petri dishes with a gentle swirling motion using a rotating tabletop shaker at low speed.

After rinsing, the samples were fixed in $1 \%$ formaldehyde diluted in PBS for 15 minutes. After three 5-minute PBS rinses, the samples were incubated for 10 minutes in $0.02 \mathrm{M}$ glycine diluted in PBS and rinsed again. Samples were post fixed in $2.5 \%$ glutaraldehyde in PBS for 30 minutes and rinsed again. Dehydration was performed through a graded ethanol series: $30 \%, 50 \%, 75 \%, 90 \%, 95 \%$ and three exchanges of $100 \%$. Samples were dried in a carbon dioxide critical point drier (Baltec CPD 030, Baltec) and then dried at room temperature.

Samples were mounted on aluminum stubs with colloidal graphite; sputter coated with gold palladium in a specified device (Baltec SCD 050, Baltec), and stored and desiccated at room temperature for 3 days.

\section{Scanning Electron Microscopy Analysis}

The photomicrograph magnification of each sample was $500 \times$ and 2,000 $\times$. All photomicrographs were obtained through a scanning electron microscope (SEM) operated at $20 \mathrm{kV}$ (Jeol JSM). After the photomicrographs were obtained, they were identified and scored as follows to verify the blood component adhesion (BCA) and to analyze the morphologic characteristics obtained in the treatment (Graph 1) (Leite et al, 2010; Leite, Moreira, Theodoro and Sampaio, 2005):

- 0 - absence of fibrin network and blood cells

- 1-scarce fibrin network and/or blood cells 
- 2-moderate fibrin network and moderate quantity of blood cells

- 3-dense fibrin network and trapped blood cells.

\section{Data Reproducibility}

The same examiner evaluated all photomicrographs and was checked for reproducibility. The photomicrographs examined three times at 7-day intervals by an operator who was blind and previously calibrated with the other two operators (kappa score $=0.93$ ). Each sample received the final score that prevailed among the three readings.

\section{Statistical Analysis}

Scores were used to verify the blood component adhesion in each group and in each sample. Results were analyzed by Kruskal-Wallis test followed by Dunn's post test $(\mathrm{p}<0.05)$ (GraphPad Prism 5.0 Software).

\section{RESULTS}

Graph 1 shows the distribution of the scores for blood component adhesion in each group and by sample. Values were expressed in raw numbers and percentage of samples that received each score.

Statistical differences are observed in Graph 2. Group 4 (cotinine + root scaling + blood) presented the highest adhesion of blood components, and group 6 (nicotine and cotinine + root scaling + nicotine and cotinine + blood $)$ had the lowest scores. The control group (group 1-PBS immersion + root scaling + blood application) presented

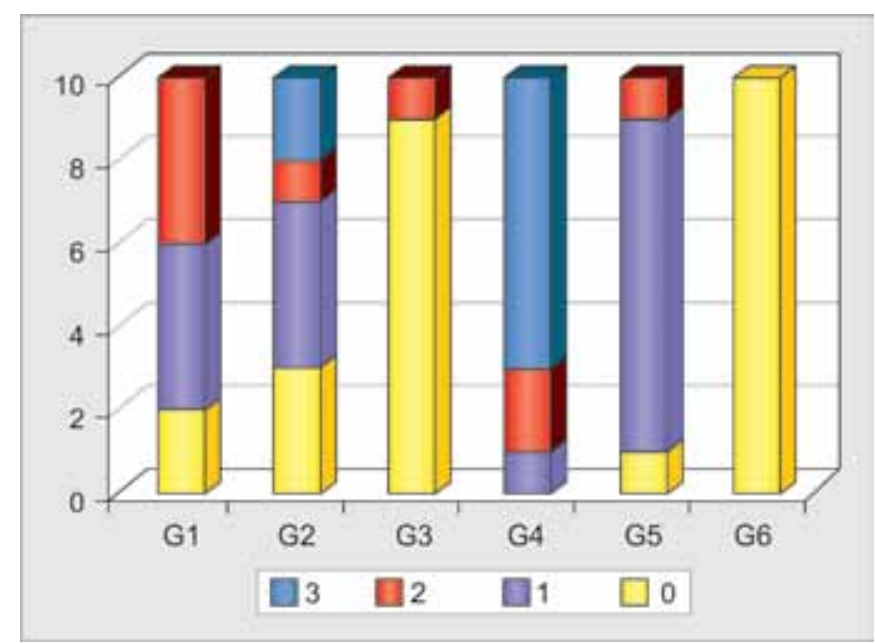

Graph 1: Score of blood component adhesion in each group: group 1 (G1): (PBS immersion + root scaling + blood application); group 2 (G2): (nicotine + root scaling + blood); group 3 (G3) (nicotine + root scaling + nicotine reapplication + blood); group 4 (G4) (cotinine + root scaling + blood); group 5(G5) (cotinine + root scaling + cotinine reapplication + blood); group 6 (G6) (nicotine and cotinine + root scaling + nicotine and cotinine + blood) blood components similar to group 2 (nicotine + root scaling + blood), 3 (nicotine + root scaling + nicotine reapplication + blood $)$ and 5 (cotinine + root scaling + cotinine reapplication+ blood). Therefore, statistically significant differences were found between groups 1 and 6 , groups 3 and 4, groups 4 and 6 (see Graph 2).

\section{DISCUSSION}

One of the most significant prejudicial factors for patients with periodontal disease is the tobacco smoking. The main mechanisms of tobacco's interference on wound healing are related with fibroblasts, platelets, and macrophages inhibition, vasoconstriction, and the overall toxicity of its nearly 4,000 components, including nicotine, carbon monoxide and cyanide. ${ }^{8,12,13}$ Cell proliferation can decrease up to $50 \%$ in cigarette smoke concentrations above $200 \mu \mathrm{g} /$ $\mathrm{ml} .{ }^{14}$ However, the direct effects of tobacco components on the blood component adhesion to root surfaces (first step of healing) were not evaluated.

The stability of the fragile attachment between the root surface and the gingiva is crucial for the outcome of periodontal healing. This first step of healing is provided by the maturation of fibrin clot. $^{3}$ Connective tissue repair on the root surface is dependent on an attachment between the fibrin clot and the root. In the absence of a stable fibrin attachment, the defect may quickly epithelialize.

Therefore, the present in vitro study evaluated the blood component adhesion in teeth impregnated with nicotine and/ or cotinine, important toxic substances present in tobacco cigarettes. Each cigarette generates $26.1 \mathrm{mg}$ of tobacco $\operatorname{tar}^{14}$

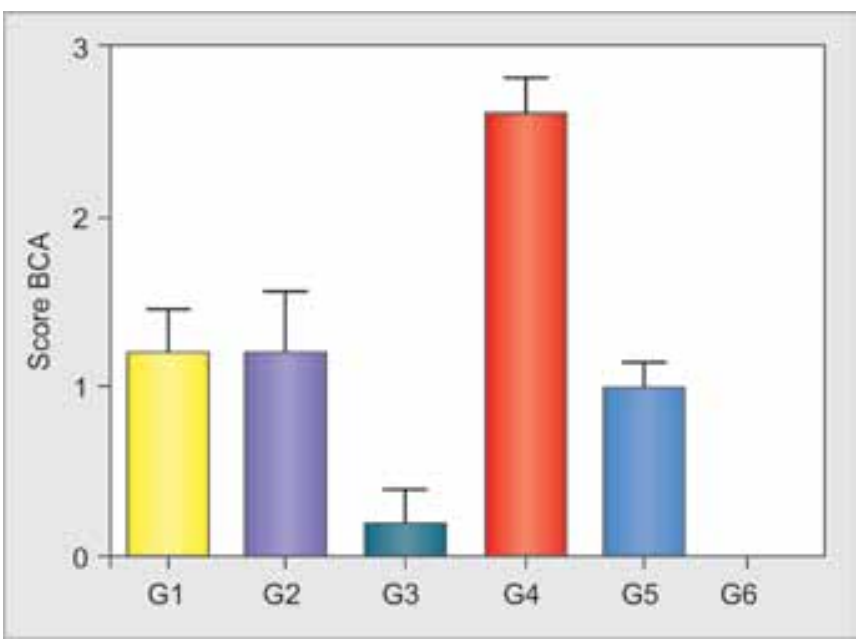

Graph 2: Column chart with mean and standard error distribution of the blood component adhesion index: group 1 (G1) (PBS immersion + root scaling + blood application); group 2 (G2) (nicotine + root scaling + blood); group 3 (G3) (nicotine + root scaling + nicotine reapplication + blood); group 4 (G4) (cotinine + root scaling + blood); group 5 (G5) (cotinine + root scaling + cotinine reapplication+ blood); group 6 (G6) (nicotine and cotinine + root scaling + nicotine and cotinine + blood). Statistically significant differences were found between groups 1 and 6 , groups 3 and 4 , groups 4 and $6(p<0.05)$ 
Considering that the saliva volume per day ranges between 1 and 1.5 liters in health individuals, tobacco concentration falls somewhere between 174 and $522 \mathrm{ug} / \mathrm{ml} /$ cigarette. Some studies showed that nicotine and cotinine concentration in human saliva has peaks of $1 \mathrm{mg} / \mathrm{mL}$ for nicotine and $1 \mu \mathrm{g} / \mathrm{ml}$ for cotinine. ${ }^{10,15}$ The concentrations employed in this study were based on these values. Despite of the great toxicity of nicotine, it has a short half-life (30 mins). Nicotine is rapidly metabolized into cotinine. ${ }^{10}$ There is variability in relation to nicotine and cotinine toxicity, and this variation can be due to low-dose or high-dose exposure to cigarette smoke. ${ }^{8}$ There is a clear indicator that, at moderate to high doses, fibroblast activity is significantly diminished, although cotinine presents molecular similarity with nicotine and can be found in high concentrations in saliva and gingival crevicular fluid, it does not affect fibroblast attachment and morphology as much as nicotine. ${ }^{10}$

In control group, samples were kept in PBS. ${ }^{16}$ The photomicrographs showed scarce fibrin network and/or blood cells (score 1) and moderate fibrin network and moderate quantity of blood cells (score 2).

The higher amount of blood components (score 3) was present in teeth exposed to cotinine (group 4). Cotinine in the concentration employed in this study was able to provoke higher blood components adhesion in relation to control group, suggesting an important effect in clot stabilization. Martinez et al $(2005)^{10}$ also suggested a benefic effect on the adhesion and proliferation of fibroblasts when teeth were exposed to cotinine. The authors observed that the adhesion and proliferation of fibroblasts were inhibited by cotinine only in concentrations greater than $10 \mu \mathrm{g} / \mathrm{ml}$, which is far greater than the highest concentration found in vivo (approximately $1 \mu \mathrm{g} / \mathrm{ml}$ ), and surprisingly, the non-scaled specimens presented greater cell density than the scaled ones.

Studies have already showed that nicotine in lower concentration is able to accelerate angiogenesis and promote wound healing, demonstrating a potential for nicotine to aid in the wound healing process. ${ }^{17}$ In the present study, when control group (group 1) is compared to groups receiving nicotine (groups 2 and 3), lower blood components are found in samples that receive reapplication of the toxic substance (group 3). The group 2, shows adhesion of blood components similar to control group (group 1), demonstrating that scaling can reduce the concentration of the nicotine on root surface and to facilitate the blood components adhesion. The fact demonstrated by Morimoto et al, ${ }^{17}$ nicotine in low concentration is able to promote healing, may be explained in part by no differences found between groups 1 and 2 results. However, the relationship between nicotine and cotinine with wound healing favoring the repair is not well explained yet. ${ }^{18}$
Greater amount of blood components also were observed on dentine surface of the groups submitted to scaling and blood application (G2 and G4) in comparison with groups that received reapplication of toxic substances after scaling (G3, G5 and G6). Although the difference was not significant statistically, the tendency of the results suggests that the periodontal treatment can be important to reduce the amount of toxic substances on root surface, allowing a better periodontal healing in low concentrations of nicotine and cotinine. This fact is mainly important for patients that quit smoking.

Statistically significant differences only were showed among groups 1(control group) and 6 (nicotine and cotinine + root scaling + nicotine and cotinine + blood), groups 3 (nicotine + root scaling + nicotine reapplication + blood $)$ and 4 (cotinine + root scaling + blood), groups 4 and 6 . The results show greater toxic effect on root dentin surface after the exposure to nicotine and cotinine. Despite the study tested, only two toxic substances of the tobacco cigarette, we can observe that the association of these substances can be harmful to the first step of periodontal healing even at low concentrations (score 0 ).

Samples only kept in nicotine or cotinine showed similar adhesion when compared with control group (PBS). Nevertheless, the present study considered the nicotine and cotinine concentrations found in human saliva, according to Morozumi et al, ${ }^{19}$ the concentration in human gingival fluid might have peaks five times higher than in human saliva.

Therefore, the study shows important information on the role of cotinine and nicotine direct effects on blood components adhesion to root surfaces. Nevertheless, the present study is an in vitro, thus, the evidences cannot be extrapolated to clinics, since the cigarette presents more than 4,000 toxic substances; nicotine and cotinine are only two of them. More studies are necessary on the effects of cigarette tar on human root surface and its effects on periodontal repair.

\section{CONCLUSION}

Wound healing is the cumulative result of clot adhesion, cell migration and division and the synthesis of extracellular molecules. An alteration in one of these steps may lead to delayed healing. The results presented in this study in vitro suggest that periodontal healing may be delayed in smokers due to the direct inhibition of clot stabilization on the root surface when nicotine and cotinine are present concomitantly.

\section{REFERENCES}

1. Polson AM, Frederick GT, Ladenheim S, Hanes PJ. The production of a root surface smear layer by instrumentation and 
its removal by citric acid. Journal of Periodontology 1984;55: 443-446.

2. Larjava H, Salonen J, Häkkinen L, Närhi T. Effect of citric acid treatment on the migration of epithelium on root surfaces in vitro. Journal of Periodontology 1988;59:95-99.

3. Wikesjö UME, Nilveus R, Selvig KA. Significance of early healing events on periodontal repair: a review. Journal of Periodontology 1992;63:158-165.

4. Baker DL, Pavlow SSA, Wikesjö UME. Fibrin clot adhesion to dentin conditioned with protein constructs: an in vitro proofof-principle study. Journal of Clinical Periodontology 2005;32: 561-566.

5. Leite FRM, Sampaio JEC, Zandim DL, Dantas AAR, Leite ERM, Leite AA. Influence of root-surface conditioning with acid and chelating agents on clot stabilization. Quintessence International 2010;41:341-349.

6. Gamal AY, Bayomy MM. Effect of cigarette smoking on human PDL fibroblasts attachment to periodontally involved root surfaces in vitro. Journal of Clinical Periodontology 2002;29: 763-770.

7. Mosely LH, Finseth F. Cigarette smoking: Impairment of digital blood flow and wound healing in the hand. The Hand 1977;9: 97-101.

8. Garg AK. Tobacco use and the Dental Implant Patient. Dental Implant Update 2010;121(1):1-5.

9. Chang Y, Huang F, Tai K, Yang L, Chou M. Mechanisms of cytotoxicity of nicotine in human periodontal ligament fibroblast cultures in vitro. Journal of Periodontal Research 2000;37: 279-285.DOI: 10.1034/j.1600-0765.2002.01612.

10. Martinez AET, Silverio KG, Fogo JC, Kirkwood KL, Rossa Jr, C. Root surface conditioning with nicotine or cotinine reduces viability and density of fibroblasts in vitro. Clinical Oral Investigation 2005;9:180-186.

11. Theodoro LH, Sampaio JEC, Haypek P, Bachmann L, Zezel DM, Garcia VG. Effect of Er:YAG and diode lasers on the adhesion of blood components and on the morphology of irradiated root surfaces. Journal of Periodontal Research 2006;41:381-390.

12. Yamamoto Y, Nishida N, Tanaka M, Hayashi N, Matsuse R, Nakayama K, Morimoto K, Shizukuishi S. Association between passive and active smoking evaluated by salivary cotinine and periodontitis. Journal of Periodontology 2005;32:1041-1046.

13. Balaji SM. Tobacco smoking and surgical healing of oral tissues: a review. Indian Journal of Dental Research 2008;19(4):344-348.

14. Zhang W, Song F, Windsor J. Cigarette smoke condensate affects the collagen-degrading ability of human gingival fibroblasts. Journal of Periodontal Research 2009;44:704-713.

15. Cuff MJ, McQuade MJ, Scheidt MJ, Sutherland DE, Van Dyke TE. The presence of nicotine on root surfaces of periodontally diseased teeth in smokers. Journal of Periodontology 1989;60: 564-569.

16. Nociti Jr FH, Nogueira-Filho GR, Tramontina VA, Machado MAN, Barros SP, Sallum EA, Sallum AW. Histometric evaluation of the effect of nicotine administration on periodontal breakdown: an in vivo study. Journal of Periodontal Research 2001;36: 361-366.

17. Morimoto N, Takemoto S, Kawazoe T, Suzuki S. Nicotine at a low concentration promotes wound healing. Journal of Surgical Research 2010;145(2):199-204.

18. Martin JW, Mousa SS, Shaker O, Mousa SA. The multiple faces of nicotine and its implications in tissue and wound repair. Experimental Dermatology 2009;18:497-505.

19. Morozumi T, Kubota T, Sato T, Okuda K, Yoshie H. Smoking cessation increases gingival blood flow and gingival crevicular fluid. Journal of Clinical Periodontology 2004;31(4):267-272.

\section{ABOUT THE AUTHORS}

\section{Shelon Cristina Souza Pinto}

Professor, Department of Dentistry, State University of Ponta Grossa Ponta Grossa, PR, Brazil

Correspondence Address: 1680, Humaitá, Araraquara, Sao Paulo Brazil, Phone: +551633016300, e-mail: shelonsouzap@gmail.com

\section{Fabio Renato Manzolli Leite}

Professor, Department of Semiology and Clinic, Federal University of Pelotas, Pelotas, RS, Brazil

\section{Lucas Amaral Fontanari}

Student, Department of Oral Diagnosis and Surgery, Araraquara Dental School, UNESP, Brazil

\section{Rodrigo Cavassim}

Student, Department of Oral Diagnosis and Surgery, Araraquara Dental School, UNESP, Brazil

\section{Amauri Antiquera Leite}

Professor, Department of Pharmacy, Araraquara Dental School UNESP, Brazil

\section{Matheus Coelho Bandéca}

Professor, Department of Postgraduation in Dentistry, CEUMA University, São Luis, Brazil

\section{Alvaro Henrique Borges}

Professor, Program in Integrated Dental Sciences, University of Cuiabá, Cuiabá, MT, Brazil

\section{José Eduardo Cezar Sampaio}

Professor, Department of Oral Diagnosis and Surgery, Araraquara Dental School, UNESP, Brazil 